\title{
MULTIPLE PERIODIC SOLUTIONS OF $p$-LAPLACIAN EQUATION WITH ONE-SIDE NAGUMO CONDITION
}

\author{
Jian Jun Zhang, Wen Bin Liu, Jin Bo Ni, and Tai Yong Chen
}

\begin{abstract}
In this paper, the existence and multiplicity of solution of periodic solutions of $p$-Laplacian boundary value problem are studied by using degree theory and upper and lower solutions method. Some known results are improved.
\end{abstract}

\section{Introduction}

In this paper, we discuss the existence of multiple solutions of the following $p$-Laplacian periodic boundary value problems:

$$
\begin{aligned}
\left(\phi_{p}\left(u^{\prime}\right)\right)^{\prime} & =f\left(t, u, u^{\prime}\right), \quad t \in[0, T], \\
u(0) & =u(T), u^{\prime}(0)=u^{\prime}(T),
\end{aligned}
$$

where $\phi_{p}(s)=|s|^{p-2} s, p>1$. Obviously, if $p=2$, Eq.(1) reduces to

$$
u^{\prime \prime}=f\left(t, u, u^{\prime}\right), \quad t \in[0, T] .
$$

In recent years, by using degree theory and upper and lower solutions method, many important results relative to Eq.(1) with certain boundary conditions have been obtained (see [2], [3], [5], [6], [7], [8], [9], [10] and references therein). By using upper and lower solutions method, [11], [12], [13], discussed the multiplicity of the periodic boundary value problems (3), (2). But for $p \neq 2$, the results of existence of periodic solutions of (1), (2) were relatively few, since the method of coincidence degree for linear operator cannot be applied directly in this case.

Motivated by the above works, we apply the method of two pairs of lower and upper solutions to discuss the solvability of the BVP (1), (2). Under the condition that $f(t, u, v)$ satisfies a one-side Nagumo condition, we obtain the

Received October 24, 2006.

2000 Mathematics Subject Classification. Primary 34C25.

Key words and phrases. p-Laplacian equations, periodic solution, one-side Nagumo condition, multiplicity, upper and lower solutions.

This work was financially supported by National Natural Science Foundation of China (10771212) and Science Foundation of China University of Mining and Technology (2006A042). 
existence of three solutions by using Leray-Schauder degree theory [1]. Our results extend some known results ([11], [12], [13], [4]).

\section{Background, notation and definitions}

In the following, we shall use the spaces $C_{T}=\{x: x \in C([0, T], \mathbb{R}), x(0)=$ $x(T)\}, C_{T}^{1}=\left\{x: x \in C^{1}([0, T], \mathbb{R}), x(0)=x(T), x^{\prime}(0)=x^{\prime}(T)\right\}$ and denote the norm in $C_{T}$ by $\|\cdot\|_{\infty}$. For $u(t) \in C_{T}^{1}$, define

$$
\|u\|=\max \left\{\|u\|_{\infty},\left\|u^{\prime}\right\|_{\infty}\right\} .
$$

Let now $H: C_{T} \longrightarrow C([0, T], \mathbb{R})$ be defined by

$$
H(h)(t)=\int_{0}^{t} h(s) d s, h \in C_{T} .
$$

For fixed $l(t) \in C([0, T], \mathbb{R})$, let us define

We have the following lemma.

$$
G_{l}(a):=\frac{1}{T} \int_{0}^{T} \phi_{p}^{-1}(a+l(t)) d t, \forall a \in \mathbb{R} .
$$

Lemma 1 ([9]). The operator $G_{l}$ has the following properties:

$\left(A_{1}\right)$ For any fixed $l(t) \in C([0, T], \mathbb{R})$ the equation

$$
G_{l}(a)=0
$$

has a unique solution $\tilde{a}(l)$;

$\left(A_{2}\right)$ The operator $\tilde{a}: C([0, T], \mathbb{R}) \rightarrow \mathbb{R}$ is continuous and sends bounded sets into bounded sets.

Let now $a: C_{T} \longrightarrow \mathbb{R}$ be defined by

$$
a(h)=\tilde{a}(H(h)), h \in C_{T} .
$$

Then it is clear that $a$ is a continuous function which sends bounded sets of $C_{T}$ into bounded sets of $\mathbb{R}$, and hence it is a completely continuous mapping.

Let us define the projectors $P, Q$ respectively by

$$
\begin{gathered}
P: C_{T} \longrightarrow C_{T}, \quad(P x)(t)=x(0), \\
Q: C_{T} \longrightarrow C_{T}, \quad(Q x)(t)=\frac{1}{T} \int_{0}^{T} x(s) d s,
\end{gathered}
$$

and the operator $\mathcal{K}: C_{T} \rightarrow C_{T}^{1}$,

$$
\mathcal{K}(h)(t):=H\left\{\phi_{p}^{-1}[a((I-Q) h)+H((I-Q) h)]\right\}(t) .
$$

And we introduce the concept of upper and lower solutions of (1), (2) and the Nagumo condition we use forward.

Definition 1. A function $\alpha(t) \in C^{1}[0, T], \phi_{p}\left(\alpha^{\prime}\right) \in C^{1}[0, T]$ is called a lower solution of (1), (2), if

$$
\begin{array}{r}
\left(\phi_{p}\left(\alpha^{\prime}\right)\right)^{\prime} \geq f\left(t, \alpha, \alpha^{\prime}\right), t \in[0, T], \\
\alpha(0)=\alpha(T), \alpha^{\prime}(0) \geq \alpha^{\prime}(T) .
\end{array}
$$

If the inequality (4) is strict, then $\alpha(t)$ is called a strict lower solution. 
Definition 2. A function $\beta(t) \in C^{1}[0, T], \phi_{p}\left(\beta^{\prime}\right) \in C^{1}[0, T]$ is called an upper solution of (1), (2), if

$$
\begin{aligned}
\left(\phi_{p}\left(\beta^{\prime}\right)\right)^{\prime} & \leq f\left(t, \beta, \beta^{\prime}\right), t \in[0, T], \\
\beta(0) & =\beta(T), \beta^{\prime}(0) \leq \beta^{\prime}(T) .
\end{aligned}
$$

If the inequality (6) is strict, then $\beta(t)$ is called a strict upper solution.

Definition 3. Given a subset $E \in[0, T] \times \mathbb{R}^{2}$, a continuous function $f$ : $[0, T] \times \mathbb{R}^{2} \longrightarrow \mathbb{R}$ is said to satisfy one-side Nagumo condition in $E$, if there exists $\varphi \in C\left(\mathbb{R}^{+},[k,+\infty)\right)$, with $k>0$, such that

$$
f(t, u, v) \leq \varphi(|v|)(1+|v|), \forall(t, u, v) \in E,
$$

and

where $\frac{1}{p}+\frac{1}{q}=1$.

$$
\int_{1}^{+\infty} \frac{1}{\varphi\left(\phi_{q}(s)\right)} d s=\infty
$$

\section{Existence results}

Theorem 1. Assume that $f:[0, T] \times \mathbb{R}^{2} \longrightarrow \mathbb{R}$ is continuous and

$\left(H_{1}\right)$ there exist an upper solution $\beta(t)$ and a lower solution $\alpha(t)$ of $B V P$ (1), (2) such that $\alpha(t) \leq \beta(t)$ for $t \in[0, T]$;

$\left(H_{2}\right) f(t, u, v)$ satisfies one-sided Nagumo condition in $E$, where

$$
E=\left\{(t, u, v) \in[0, T] \times \mathbb{R}^{2}: \alpha(t) \leq u \leq \beta(t)\right\} .
$$

Then there exists a solution $u(t)$ of $B V P(1)$, (2) satisfying $\alpha(t) \leq u(t) \leq$ $\beta(t)$ for $t \in[0, T]$.

Remark 1. It is easy to see that the conditions in [4] are greater than the conditions of Theorem 1.

In order to prove the Theorem 1, we consider the auxiliary boundary value problem

$$
\begin{gathered}
\left(\phi_{p}(u)\right)^{\prime}=\lambda f\left(t, u, u^{\prime}\right), \quad \lambda \in(0,1), \\
u(0)=u(T), \quad u^{\prime}(0)=u^{\prime}(T) .
\end{gathered}
$$

Lemma 2 ([9]). Let $\Omega \subset C^{1}[0, T]$ be an open bounded set. Assume that

$\left(B_{1}\right)$ the problem (8), (9) has no solution on $\partial \Omega$ for $0<\lambda<1$;

$\left(B_{2}\right)$ the equation

$$
F(s):=\frac{1}{T} \int_{0}^{T} f(t, s, 0) d t=0
$$

has no solution on $\partial \Omega \cap \mathbb{R}$;

$\left(B_{3}\right)$ the Brouwer degree

$$
\operatorname{deg}_{B}(F, \Omega \cap \mathbb{R}, 0) \neq 0 .
$$


Then the problem (1), (2) has a solution in $\bar{\Omega}$.

Proof of Theorem 1. For $(t, u, v) \in[0, T] \times \mathbb{R}^{2}$ define

(10) $\bar{f}(t, u, v)= \begin{cases}f(t, \beta(t), v)+u-\beta(t) & \text { for } u>\beta(t), \\ f(t, u, v) & \text { for } \alpha(t) \leq u \leq \beta(t), \\ f(t, \alpha(t), v)+u-\alpha(t) & \text { for } u<\alpha(t) .\end{cases}$

The modified problem corresponding to $(1),(2)$ is

$$
\begin{gathered}
\left(\phi_{p}\left(u^{\prime}\right)\right)^{\prime}=\bar{f}\left(t, u, u^{\prime}\right), \quad t \in[0, T], \\
u(0)=u(T), \quad u^{\prime}(0)=u^{\prime}(T) .
\end{gathered}
$$

In order to use Lemma 2, we consider the homotopy problem of BVP (11), (12)

$$
\begin{gathered}
\left(\phi_{p}\left(u^{\prime}\right)\right)^{\prime}=\lambda \bar{f}\left(t, u, u^{\prime}\right), \quad \lambda \in(0,1), \\
u(0)=u(T), \quad u^{\prime}(0)=u^{\prime}(T) .
\end{gathered}
$$

First, we can claim that there exists a constant $M_{1}>0$ such that $\|u\|_{\infty}<$ $M_{1}, \lambda \in(0,1)$, where $u(t)$ is a possible solution of BVP (13), (14), and $M_{1}$ is large enough such that

$$
\begin{gathered}
f(t, \beta(t), 0)+M_{1}-\beta(t)>0, \quad M_{1}>\beta(t) \text { for } t \in[0, T], \\
f(t, \alpha(t), 0)-M_{1}-\alpha(t)<0, \quad-M_{1}<\alpha(t) \text { for } t \in[0, T] .
\end{gathered}
$$

Otherwise, there exists a point $t_{0} \in[0, T)$ such that $u\left(t_{0}\right)=\min _{t \in[0, T]} u(t) \leq$ $-M_{1}$ or $u\left(t_{0}\right)=\max _{t \in[0, T]} u(t) \geq M_{1}$. Without loss of generality, assume that $u\left(t_{0}\right)=\max _{t \in[0, T]} u(t) \geq M_{1}$. There are two cases as following:

Case 1. If $t_{0} \in(0, T)$, then $u^{\prime}\left(t_{0}\right)=0$ and

$$
\begin{aligned}
\left(\phi_{p}\left(u^{\prime}\left(t_{0}\right)\right)\right)^{\prime} & =\lambda\left(f\left(t_{0}, \beta\left(t_{0}\right), 0\right)+u\left(t_{0}\right)-\beta\left(t_{0}\right)\right) \\
& \geq \lambda\left(f\left(t_{0}, \beta\left(t_{0}\right), 0\right)+M_{1}-\beta\left(t_{0}\right)\right)>0 .
\end{aligned}
$$

So, there exists a constant $\delta>0$ such that $\left(\phi_{p}\left(u^{\prime}(t)\right)\right)^{\prime}>0$ for $t \in\left(t_{0}, t_{0}+\delta\right)$. This implies that $\phi_{p}\left(u^{\prime}(t)\right)$ is increasing on $\left(t_{0}, t_{0}+\delta\right)$. Thus

$$
\phi_{p}\left(u^{\prime}(t)\right)>\phi_{p}\left(u^{\prime}\left(t_{0}\right)\right)=\phi_{p}(0)=0, \quad t \in\left(t_{0}, t_{0}+\delta\right),
$$

which shows $u^{\prime}(t)>0, t \in\left(t_{0}, t_{0}+\delta\right)$ from the monotonicity of $\phi_{p}$. Namely, $u(t)$ is increasing on $\left(t_{0}, t_{0}+\delta\right)$, contradicting $u\left(t_{0}\right)=\max _{t \in[0,1]} u(t)$.

Case 2. If $t_{0}=0$, then

$$
u^{\prime}(0) \leq 0, u^{\prime}(T) \geq 0 .
$$

From (14), we know that $u^{\prime}(0)=0$. A similar argument concludes a contradiction with $u(0)=\max _{t \in[0,1]} u(t)$.

Second, we shall prove that there exists $M_{2}>0$ such that $\left\|u^{\prime}\right\|_{\infty} \leq M_{2}$.

Noting the definition of $\bar{f}$, the condition $\left(H_{2}\right)$ implies that

$$
\left(\phi_{p}\left(u^{\prime}(t)\right)^{\prime} \leq \varphi\left(\left|u^{\prime}\right|\right)\left(1+\left|u^{\prime}\right|\right)+2 M_{1}, t \in[0, T] .\right.
$$

From $u(0)=u(T), u^{\prime}(0)=u^{\prime}(T)$, we can find $t_{0} \in[0, T)$ such that $u^{\prime}\left(t_{0}\right)=0$. Without loss of generality, we assume that $t_{0} \in(0, T)$. 
By the definition of Nagumo condition, it follows that there exist $M_{2}, M_{3} \in$ $(1, \infty)$ with $M_{3}<M_{2}$, such that

$$
\begin{gathered}
\int_{1}^{\phi_{p}\left(M_{3}\right)} \frac{1}{\varphi\left(\phi_{q}(s)\right)} d s=K>2 M_{1}(2+T), \\
\int_{1}^{\phi_{p}\left(M_{2}\right)} \frac{1}{\varphi\left(\phi_{q}(s)\right)} d s>K+2 M_{1}(2+T),
\end{gathered}
$$

where $K$ is a positive constant.

Suppose that there exists $t_{1} \in\left(t_{0}, T\right]$ such that

$$
u^{\prime}\left(t_{1}\right)=\max \left\{u^{\prime}(t): t \in\left[t_{0}, T\right]\right\}=c_{1}>1 .
$$

We can find $\mu \in\left[t_{0}, t_{1}\right]$ such that $u^{\prime}(t)>1, t \in\left(\mu, t_{1}\right], u^{\prime}(\mu)=1$.

Integrating (17) over $\left[\mu, t_{1}\right]$, we get

$$
\begin{aligned}
\int_{\mu}^{t_{1}} \frac{\left(\phi_{p}\left(u^{\prime}(t)\right)\right)^{\prime}}{\varphi\left(u^{\prime}\right)} d t & \leq \int_{\mu}^{t_{1}}\left(1+u^{\prime}+\frac{2 M_{1}}{\varphi\left(u^{\prime}\right)}\right) d t \\
& \leq \int_{\mu}^{t_{1}}\left(2 u^{\prime}+2 M_{1}\right) d t \leq 2 M_{1}(2+T)
\end{aligned}
$$

Let $s=\phi_{p}\left(u^{\prime}(t)\right)$, we have

$$
\int_{1}^{\phi_{p}\left(c_{1}\right)} \frac{1}{\varphi\left(\phi_{q}(s)\right)} d s \leq 2 M_{1}(2+T) .
$$

By (18), we know that $\phi_{p}\left(c_{1}\right)<\phi_{p}\left(M_{3}\right)$. Therefore $u^{\prime}(t)<M_{3}, t \in\left[t_{0}, T\right]$, $u^{\prime}(0)<M_{3}$.

Further suppose that there exists $t_{2} \in\left[0, t_{0}\right)$ such that

$$
u^{\prime}\left(t_{2}\right)=\max \left\{u^{\prime}(t): t \in\left[0, t_{0}\right]\right\}=c_{2}>M_{3} .
$$

Then we can find $\nu \in\left[0, t_{2}\right]$ such that $u^{\prime}(t)>M_{3}, t \in\left(\nu, t_{2}\right], u^{\prime}(\nu)=M_{3}$.

Integrating (17) over $\left[\nu, t_{2}\right]$, we get

$$
\int_{\phi_{p}\left(M_{3}\right)}^{\phi_{p}\left(c_{2}\right)} \frac{1}{\varphi\left(\phi_{q}(s)\right)} d s \leq 2 M_{1}(2+T) .
$$

Combining with (18), we have

$$
\int_{1}^{\phi_{p}\left(c_{2}\right)} \frac{1}{\varphi\left(\phi_{q}(s)\right)} d s \leq K+2 M_{1}(2+T) .
$$

By (19), we know that $\phi_{p}\left(c_{2}\right)<\phi_{p}\left(M_{2}\right)$. Therefore $u^{\prime}(t)<M_{2}, t \in\left[0, t_{0}\right]$.

Thus we get

$$
u^{\prime}(t)<M_{2}, t \in[0, T] .
$$

Suppose that there exists $t_{3} \in\left[0, t_{0}\right)$ such that

$$
u^{\prime}\left(t_{3}\right)=\min \left\{u^{\prime}(t): t \in\left[0, t_{0}\right]\right\}=-c_{3}<-1 .
$$


A similar argument can concludes that $c_{3}<M_{3}$. So

$$
u^{\prime}(t)>-M_{3}, t \in\left[0, t_{0}\right], u^{\prime}(T)>-M_{3} .
$$

Suppose that there exists $t_{4} \in\left[t_{0}, T\right]$ such that

$$
u^{\prime}\left(t_{4}\right)=\min \left\{u^{\prime}(t): t \in\left[t_{0}, T\right]\right\}=-c_{4}<-M_{3} .
$$

A similar argument can concludes that $c_{4}<M_{2}$. So

$$
u^{\prime}(t)>-M_{2}, t \in\left[t_{0}, T\right] .
$$

Therefore we have

$$
u^{\prime}(t)>-M_{2}, t \in[0, T]
$$

Hence

$$
\left\|u^{\prime}\right\|_{\infty} \leq M_{2} .
$$

Next, we shall prove that the BVP (11), (12) has at least one solution by using Lemma 2.

Set

$$
\Omega_{1}=\left\{u(t) \in C_{T}^{1}:\|u\|<M_{0}\right\},
$$

where $M_{0}=\max \left\{M_{1}, M_{2}\right\}+1$.

Obviously, the hypothesis $\left(B_{1}\right)$ of Lemma 2 is satisfied.

By (15), (16), we know that

$$
\bar{f}\left(t,-M_{0}, 0\right)<0, \bar{f}\left(t, M_{0}, 0\right)>0 .
$$

Applying the monotonicity of $\phi_{p}$, we immediately get

$$
\begin{gathered}
F\left(-M_{0}\right)=\frac{1}{T} \int_{0}^{T} \bar{f}\left(t,-M_{0}, 0\right) d t<0, \\
F\left(M_{0}\right)=\frac{1}{T} \int_{0}^{T} \bar{f}\left(t, M_{0}, 0\right) d t>0 .
\end{gathered}
$$

Thus, the hypothesis $\left(B_{2}\right)$ of Lemma 2 is true.

By using the property of Brouwer degree, we see that

$$
\operatorname{deg}_{B}\left(F, \Omega_{1} \cap \mathbb{R}, 0\right)=1 .
$$

Hence, the hypothesis $\left(B_{3}\right)$ of Lemma 2 is also satisfied. By Lemma 2, it can be shown that the BVP (11), (12) has one solution $u(t)$.

Finally, we can claim that $\alpha(t) \leq u(t) \leq \beta(t)$ for $t \in[0, T]$, where $u(t)$ is a solution of BVP (11), (12). Otherwise, there exists a point $t_{1} \in[0, T)$ such that $u\left(t_{1}\right)<\alpha\left(t_{1}\right)$ or $u\left(t_{1}\right)>\beta\left(t_{1}\right)$. Without loss of generality, assume that $u\left(t_{1}\right)>\beta\left(t_{1}\right)$.

Let $x(t)=u(t)-\beta(t)$. Then there exists a point $t_{2} \in[0, T)$ such that $x\left(t_{2}\right)=\max _{t \in[0, T]} x(t)>0$. There are two cases as following: 
Case 1. If $t_{2} \in(0, T)$, then $x^{\prime}\left(t_{2}\right)=0$. For the definition of the upper solution, thus

$$
\begin{aligned}
\left(\phi_{p}\left(u^{\prime}\left(t_{2}\right)\right)-\phi_{p}\left(\beta^{\prime}\left(t_{2}\right)\right)\right)^{\prime} \geq & \bar{f}\left(t_{2}, u\left(t_{2}\right), u^{\prime}\left(t_{2}\right)\right)-f\left(t_{2}, \beta\left(t_{2}\right), \beta^{\prime}\left(t_{2}\right)\right) \\
= & f\left(t_{2}, \beta\left(t_{2}\right), u^{\prime}\left(t_{2}\right)\right)+u\left(t_{2}\right)-\beta\left(t_{2}\right) \\
& -f\left(t_{2}, \beta\left(t_{2}\right), \beta^{\prime}\left(t_{2}\right)\right) \\
= & u\left(t_{2}\right)-\beta\left(t_{2}\right)=x\left(t_{2}\right)>0 .
\end{aligned}
$$

So, there exists a constant $\varepsilon>0$ such that $\left(\phi_{p}\left(u^{\prime}(t)\right)-\phi_{p}\left(\beta^{\prime}(t)\right)\right)^{\prime}>0$ for $t \in$ $\left(t_{2}, t_{2}+\varepsilon\right)$. This implies that $\phi_{p}\left(u^{\prime}(t)\right)-\phi_{p}\left(\beta^{\prime}(t)\right)$ is increasing on $\left(t_{2}, t_{2}+\varepsilon\right)$. Thus

$$
\phi_{p}\left(u^{\prime}(t)\right)-\phi_{p}\left(\beta^{\prime}(t)\right)>\phi_{p}\left(u^{\prime}\left(t_{2}\right)\right)-\phi_{p}\left(\beta^{\prime}\left(t_{2}\right)\right)=0, \quad t \in\left(t_{2}, t_{2}+\varepsilon\right),
$$

which shows $u^{\prime}(t)>\beta^{\prime}(t), t \in\left(t_{2}, t_{2}+\varepsilon\right)$ from the monotonicity of $\phi_{p}$. Namely, $x(t)$ is increasing on $\left(t_{2}, t_{2}+\varepsilon\right)$, contradicting $x\left(t_{2}\right)=\max _{t \in[0, T]} x(t)$.

Case 2. If $t_{2}=0$, then $x^{\prime}(0)=0$ from $x(0)=x(T)=\max _{t \in[0, T]} x(t) . \mathrm{A}$ similar argument concludes a contradiction with $x(0)=\max _{t \in[0, T]} x(t)$.

From (10), we get that $u(t)$ is a solution of BVP (1), (2) satisfying $\alpha(t) \leq$ $u(t) \leq \beta(t)$ for $t \in[0,1]$.

This completes the proof of Theorem 1 .

Corollary 1. Assume that $f:[0, T] \times \mathbb{R}^{2} \longrightarrow \mathbb{R}$ is continuous and

$\left(H_{1}^{\prime}\right)$ there exist two constants $r_{1} \leq r_{2}$, such that

$$
f\left(t, r_{1}, 0\right) \leq 0 \leq f\left(t, r_{2}, 0\right) \forall t \in[0, T] ;
$$

$\left(H_{2}^{\prime}\right) f(t, u, v)$ satisfies one-side Nagumo condition in $E$, where

$$
E=\left\{(t, u, v) \in[0, T] \times \mathbb{R}^{2}: r_{1} \leq u \leq r_{2}\right\} .
$$

Then there exists a solution $u(t)$ of $B V P(1)$, (2), satisfying $r_{1} \leq u(t) \leq$ $r_{2}$ for $t \in[0, T]$.

\section{Multiplicity results}

In this section, we shall apply upper and lower solutions method and degree theory to get the following multiplicity result.

Theorem 2. Assume that $f:[0, T] \times \mathbb{R}^{2} \longrightarrow \mathbb{R}$ is continuous and

$\left(H_{3}\right)$ there exist strict upper solutions $\beta_{1}(t), \beta_{2}(t)$ and strict lower solutions $\alpha_{1}(t), \alpha_{2}(t)$ of BVP (1), (2), such that $\alpha_{1}(t)<\beta_{1}(t)<\alpha_{2}(t)<\beta_{2}(t)$ for $t \in[0, T]$.

$\left(H_{4}\right) f(t, u, v)$ satisfies one-side Nagumo condition in $E$, where

$$
E=\left\{(t, u, v) \in[0, T] \times \mathbb{R}^{2}: \alpha_{1}(t) \leq u \leq \beta_{2}(t)\right\} .
$$

Then there exist three solutions $u_{1}(t), u_{2}(t), u_{3}(t)$ of $B V P(1)$, (2) satisfying $\alpha_{1}(t)<u_{1}(t)<\beta_{1}(t), \alpha_{2}(t)<u_{2}(t)<\beta_{2}(t)$ for $t \in[0, T]$ and $u_{3}(t) \nless \beta_{1}(t)$, $u_{3}(t) \ngtr \alpha_{2}(t)$ for $t \in[0, T]$. 
In order to prove the Theorem 2, we first prove the following lemma.

Define $L_{p}: D\left(L_{p}\right) \subset C_{T}^{1} \longrightarrow L^{1}([0, T], \mathbb{R})$, by

$$
\left(L_{p} u\right)(t)=\left(\phi_{p}\left(u^{\prime}(t)\right)\right)^{\prime}
$$

where $D\left(L_{p}\right)=\left\{u: u \in C^{1}[0, T], \phi_{p}(u) \in C^{1}[0, T], u(0)=u(T), u^{\prime}(0)=u^{\prime}(T)\right\}$ and $\mathcal{G}_{f}(u, \lambda): \operatorname{dom} \mathcal{G}_{f} \subset C_{T}^{1} \times[0,1] \longrightarrow L^{1}([0, T], \mathbb{R})$, by

$$
\mathcal{G}_{f}(u, \lambda)=P u+Q N_{f}(u)+\left(\mathcal{K} \circ\left[\lambda(I-Q) N_{f}\right]\right)(u),
$$

where $\left(N_{f} u\right)(t)=f\left(t, u, u^{\prime}\right)$.

Lemma 3. Assume that $f:[0, T] \times \mathbb{R}^{2} \longrightarrow \mathbb{R}$ is continuous and

$\left(H_{5}\right)$ there exist a strict upper solution $\beta(t)$ and a strict lower solution $\alpha(t)$ of $B V P(1),(2)$, such that $\alpha(t)<\beta(t)$ for $t \in[0, T]$;

$\left(H_{6}\right) f(t, u, v)$ satisfies one-side Nagumo condition in $E$, where

$$
E=\left\{(t, u, v) \in[0, T] \times \mathbb{R}^{2}: \alpha(t) \leq u \leq \beta(t)\right\} .
$$

Then there exists a constant $M_{\alpha, \beta}$ (only depending on $\alpha, \beta$ ), such that

$$
D_{L-S}\left(I-\mathcal{G}_{f}(\cdot, 1), \Omega, \theta\right)=-1, \forall R>M_{\alpha, \beta},
$$

where $\Omega=\left\{u: u \in D\left(\mathcal{G}_{f}(\cdot, 1)\right), \alpha(t)<u(t)<\beta(t), \forall t \in[0, T],\left\|u^{\prime}\right\|_{\infty}<R\right\}$.

Proof. It follows from [9] that the operator $\mathcal{G}_{\bar{f}}(u, \lambda)$ is compact and continuous and BVP (13), (14) is equivalent to $u=\mathcal{G}_{\bar{f}}(u, \lambda), \lambda \in(0,1)$.

Using the similar argument in the proof of Theorem 1, there is a constant $M_{\alpha, \beta}$ (only depending on $\alpha, \beta$ ), such that

$$
\begin{gathered}
\|u\|_{\infty}<M_{\alpha, \beta},\left\|u^{\prime}\right\|_{\infty}<M_{\alpha, \beta}, \\
-M_{\alpha, \beta}<\alpha(t), \beta(t)<M_{\alpha, \beta}, \forall t \in[0, T], \\
\bar{f}\left(t,-M_{\alpha, \beta}, 0\right)<0, \bar{f}\left(t, M_{\alpha, \beta}, 0\right)>0, \forall t \in[0, T],
\end{gathered}
$$

where $u(t)$ is a possible solution of $u=\mathcal{G}_{\bar{f}}(u, \lambda), \lambda \in(0,1]$.

Specially,

$$
\alpha(t)<u(t)<\beta(t), \forall t \in[0, T],
$$

where $u(t)$ is a possible solution of $u=\mathcal{G}_{\bar{f}}(u, 1)$.

Set

$$
\Omega_{R}=\left\{u: u \in D\left(\mathcal{G}_{\bar{f}}\right),\|u\|<R\right\}, R>M_{\alpha, \beta} .
$$

From (23), we can get that $u \neq \mathcal{G}_{\bar{f}}(u, 0), u \in \partial \Omega_{R}$.

Thus

$$
u \neq \mathcal{G}_{\bar{f}}(u, \lambda), \lambda \in[0,1], u \in \partial \Omega_{R} .
$$

By the homotopy invariance property, we get that

$$
\begin{aligned}
D_{L-S}\left(I-\mathcal{G}_{\bar{f}}(\cdot, 1), \Omega_{R}, \theta\right) & =D_{L-S}\left(I-\mathcal{G}_{\bar{f}}(\cdot, 0), \Omega_{R}, \theta\right), \\
& =\operatorname{deg}_{B}\left(I-\mathcal{G}_{\bar{f}}(\cdot, 0), \Omega_{R} \cap \mathbb{R}, 0\right), \\
& =\operatorname{deg}_{B}\left(-\bar{F}, \Omega_{R} \cap \mathbb{R}, 0\right),
\end{aligned}
$$


where $\bar{F}$ defined by

$$
\bar{F}(a):=\frac{1}{T} \int_{0}^{T} \bar{f}(\tau, a, 0) d \tau .
$$

By (23), we know that

$$
\bar{f}(t,-R, 0)<0, \bar{f}(t, R, 0)>0, \forall t \in[0, T] .
$$

Applying the monotonicity of $\phi_{p}$, we immediately get

$$
\begin{gathered}
\bar{F}(-R)=\frac{1}{T} \int_{0}^{T} \bar{f}(\tau,-R, 0) d \tau<0, \\
\bar{F}(R)=\frac{1}{T} \int_{0}^{T} \bar{f}(\tau, R, 0) d \tau>0 .
\end{gathered}
$$

By using the property of Brouwer degree, we see that

$$
\operatorname{deg}_{B}\left(-\bar{F}, \Omega_{R} \cap \mathbb{R}, 0\right)=-1 .
$$

Thus

$$
D_{L-S}\left(I-\mathcal{G}_{\bar{f}}(\cdot, 1), \Omega_{R}, \theta\right)=-1 .
$$

By (24), we know that

$$
D_{L-S}\left(I-\mathcal{G}_{f}(\cdot, 1), \Omega, \theta\right)=-1 .
$$

This completes the proof of Lemma 3 .

Proof of Theorem 2. By assumption $H_{3}$ and Lemma 3, there exists a constant $R$, such that

$$
\begin{aligned}
D_{L-S}\left(I-\mathcal{G}_{f}(\cdot, 1), \Omega_{1}, \theta\right) & =D_{L-S}\left(I-\mathcal{G}_{f}(\cdot, 1), \Omega_{2}, \theta\right) \\
& =D_{L-S}\left(I-\mathcal{G}_{f}(\cdot, 1), \Omega_{3}, \theta\right)=-1
\end{aligned}
$$

where

$$
\begin{aligned}
& \Omega_{1}=\left\{u: u \in D\left(\mathcal{G}_{f}\right), \alpha_{1}(t)<u(t)<\beta_{1}(t), \forall t \in[0, T],\left\|u^{\prime}\right\|_{\infty}<R\right\}, \\
& \Omega_{2}=\left\{u: u \in D\left(\mathcal{G}_{f}\right), \alpha_{2}(t)<u(t)<\beta_{2}(t), \forall t \in[0, T],\left\|u^{\prime}\right\|_{\infty}<R\right\}, \\
& \Omega_{3}=\left\{u: u \in D\left(\mathcal{G}_{f}\right), \alpha_{1}(t)<u(t)<\beta_{2}(t), \forall t \in[0, T],\left\|u^{\prime}\right\|_{\infty}<R\right\} .
\end{aligned}
$$

Because $\Omega_{1}, \Omega_{2}$ and $\Omega_{3} \backslash \overline{\Omega_{1} \cup \Omega_{2}}$ are mutually disjoint and $\Omega_{3}=\Omega_{1} \cup \Omega_{2} \cup$ $\Omega_{3} \backslash \overline{\Omega_{1} \cup \Omega_{2}}$, we can get

$$
\begin{aligned}
& D_{L-S}\left(I-\mathcal{G}_{f}(\cdot, 1), \Omega_{3} \backslash \overline{\Omega_{1} \cup \Omega_{2}}, \theta\right) \\
= & D_{L-S}\left(I-\mathcal{G}_{f}(\cdot, 1), \Omega_{3}, \theta\right) \\
& -D_{L-S}\left(I-\mathcal{G}_{f}(\cdot, 1), \Omega_{1}, \theta\right)-D_{L-S}\left(I-\mathcal{G}_{f}(\cdot, 1), \Omega_{2}, \theta\right) \\
= & 1 \neq 0 .
\end{aligned}
$$

The above discussion implies that the operator equation $u=\mathcal{G}_{f}(u, 1)$, that is $\operatorname{BVP}(1),(2)$ have at least one solution in the set $\Omega_{1}, \Omega_{2}$ and $\Omega_{3} \backslash \overline{\Omega_{1} \cup \Omega_{2}}$, respectively. That is, there exist three solutions $u_{i}(t), i=1,2,3$, such that

$$
\alpha_{1}(t)<u_{1}(t)<\beta_{1}(t), \forall t \in[0, T]
$$




$$
\begin{gathered}
\alpha_{2}(t)<u_{2}(t)<\beta_{2}(t), \forall t \in[0, T], \\
u_{3}(t) \nless \beta_{1}(t), u_{3}(t) \nsupseteq \alpha_{2}(t), \forall t \in[0, T] .
\end{gathered}
$$

This completes the proof of Theorem 2 .

Corollary 2. Assume that $f:[0, T] \times \mathbb{R}^{2} \longrightarrow \mathbb{R}$ is continuous and $\left(H_{3}^{\prime}\right)$ there exist $2 m$ constants $c_{i}, d_{i}, i=1,2, \ldots, m$, with

$$
c_{1}<d_{1}<c_{2}<d_{2}<\cdots<c_{m}<d_{m},
$$

such that

$$
f\left(t, c_{i}, 0\right)<0<f\left(t, d_{i}, 0\right), i=1,2, \ldots, m, \forall t \in[0, T] ;
$$

$\left(H_{4}^{\prime}\right) f(t, u, v)$ satisfies one-side Nagumo condition in $E$, where

$$
E=\left\{(t, u, v) \in[0,1] \times \mathbb{R}^{2}: c_{1} \leq u \leq d_{m}\right\} .
$$

Then there exist $2 m-1$ solutions $u_{i}(t), i=1,2, \ldots, 2 m-1$ of $B V P(1),(2)$.

Example. Consider the following boundary value problem

$$
\begin{aligned}
\left(\phi_{p}\left(u^{\prime}\right)\right)^{\prime} & =f\left(t, u, u^{\prime}\right), t \in[0, T], \\
u(0) & =u(T), u^{\prime}(0)=u^{\prime}(T),
\end{aligned}
$$

where $f\left(t, u, u^{\prime}\right)=\sin (u)|u|^{m}+u^{r}\left(u^{\prime}\right)^{p}+\left(u^{\prime}\right)^{k}-1$ and $m, r, k$ are positive constants with $k \leq p$.

Obviously, $f\left(t, u, u^{\prime}\right)$ satisfies one-side Nagumo condition and there are infinite constants $\left(2 n \pm \frac{1}{2}\right) \pi, n \in \mathbb{Z}$, such that

$$
f\left(t,\left(2 n-\frac{1}{2}\right) \pi, 0\right)<0<f\left(t,\left(2 n+\frac{1}{2}\right) \pi, 0\right), t \in[0, T] .
$$

By using Corollary 2, we conclude that BVP (25), (26) exists infinite solutions.

Acknowledgements. The authors would like to thank the referee for his (or her) valuable suggestions.

\section{References}

[1] K. Deimling, Nonlinear Functional Analysis, Springer-Verlag, Berlin, 1985.

[2] M. Del Pino, M. Elgueta, and R. Manásevich, A homotopic deformation along $p$ of a Leray-Schauder degree result and existence for $\left(\left|u^{\prime}\right|^{p-2} u^{\prime}\right)^{\prime}+f(t, u)=0, u(0)=u(T)=$ 0, $p>1$, J. Differential Equations 80 (1989), no. 1, 1-13.

[3] M. García-Huidobro, C. P. Gupta, and R. Manásevich, Solvability for a nonlinear threepoint boundary value problem with p-Laplacian-like operator at resonance, Abstr. Appl. Anal. 6 (2001), no. 4, 191-213.

[4] A. Granas, R. B. Guenther, and J. W. Lee, Some general existence principles in the Caratheodory theory of nonlinear differential systems, J. Math. Pures Appl. (9) 70 (1991), no. 2, 153-196.

[5] D. Jiang and W. Gao, Upper and lower solution method and a singular boundary value problem for the one-dimensional p-Laplacian, J. Math. Anal. Appl. 252 (2000), no. 2, 631-648.

[6] D. Jiang and J. Wang, A generalized periodic boundary value problem for the onedimensional p-Laplacian, Ann. Polon. Math. 65 (1997), no. 3, 265-270. 
[7] L. Lian and W. Ge, The existence of solutions of m-point p-Laplacian boundary value problems at resonance, Acta Math. Appl. Sin. 28 (2005), no. 2, 288-295.

[8] B. Liu and J. Yu, Existence of solutions for the periodic boundary value problems with p-Laplacian operator, J. Systems Sci. Math. Sci. 23 (2003), no. 1, 76-85.

[9] R. Manásevich and J. Mawhin, Periodic solutions for nonlinear systems with $p$ Laplacian-like operators, J. Differential Equations 145 (1998), no. 2, 367-393.

[10] D. O'regan, Some general existence principles and results for $\left(\phi\left(y^{\prime}\right)\right)^{\prime}=q f\left(t, y, y^{\prime}\right), 0<$ $t<1$, SIAM J. Math. Anal. 24 (1993), no. 3, 648-668.

[11] I. Rachünková, Upper and lower solutions and topological degree, J. Math. Anal. Appl. 234 (1999), no. 1, 311-327.

[12] _ Upper and lower solutions and multiplicity results, J. Math. Anal. Appl. 246 (2000), no. 2, 446-464.

[13] X. Yang, Multiple positive solutions of second-order differential equations, Nonlinear Anal. 62 (2005), no. 1, 107-116.

JiAn JUN Zhang

Department of Mathematics

China University of Mining and Technology

Xuzhou Jiangsu 221008, P. R. China

E-mail address: zjj-cumt@163.com

Wen Bin LiU

Department of Mathematics

China University of Mining and Technology

Xuzhou Jiangsu 221008, P. R. China

E-mail address: wblium@163.com

JiN Bo Ni

Department of Mathematics

Anhui University of Science and Technology

Huinan Anhui 232001, P. R. China

E-mail address: nijinbo2005@126.com

Tai Yong Chen

Department of Mathematics

China University of Mining and Technology

Xuzhou Jiangsu 221008, P. R. China

E-mail address: ctycumt@163.com 